REVESCO. Revista de Estudios Cooperativos

ISSN: $1885-8031$

http://dx.doi.org/10.5209/REVE.61935

\title{
Banca Ética y Banca Tradicional. Comparativa entre Triodos Bank y Banco Santander
}

\author{
Francisco José Climent Diranzo ${ }^{1}$ y María Vicenta Escrivá Llidó ${ }^{2}$
}

Recibido: 26 de julio de 2017 / Aceptado: 11 de septiembre de 2018

Resumen. La crisis financiera de 2008 ha provocado cambios en la estructura bancaria tradicional, generando una desconfianza por parte de los ciudadanos con el sistema financiero tradicional, como consecuencia de ello ha surgido otro tipo de banca, la Banca Ética. El objetivo de este trabajo es estudiar si la Banca Ética puede llegar a ser igual de rentable que la Banca Tradicional, invirtiendo principalmente en valores sociales. Para ello, se realiza un análisis comparativo entre la Banca Ética (Triodos Bank) y la Banca Tradicional (Banco Santander). Para alcanzar el objetivo propuesto se realiza un análisis económico centrado en la actividad financiera de ambas tipologías de bancos durante el período 2011-2017 y se analizan el Balance de Situación, la Cuenta de Pérdidas y Ganancias y las Ratios de Liquidez, Endeudamiento y Rentabilidad de ambos bancos. Se comprueba que la Banca Ética no llega a ser tan rentable como la Banca tradicional, pero ha conseguido atraer a los clientes con sus inversiones sociales y la transparencia total de sus acciones financieras.

Palabras clave: Banca Ética; Banca Tradicional; Banca Social; Economía Social y Valores Sociales. Claves Econlit: G2; A2; M4.

\section{[en] Ethical Banking and Conventional Banking. Triodos Bank vs Banco Santander}

\begin{abstract}
The financial crisis of 2008 has caused changes in the traditional banking structure, generating a distrust on the part of citizens with the traditional financial system, as a result of this has emerged another type of banking, Ethical Banking. The objective of this paper is to study if the Ethical Banking can be as profitable as the Conventional Banking, investing mainly in social values. For this purpose, a comparative analysis is carried out between the Ethical Banking (Triodos Bank) and the Conventional Bank (Banco Santander). To achieve the proposed objective, an economic analysis is carried out focused on the financial activity of both types of banks during 2011-2017 and the Balance Sheet, the Profit and Loss Account and the Liquidity, Indebtedness and Profitability Ratios are analyzed. Results shows that the Ethical Banking does not become as profitable as Conventional Banking, but it has managed to attract customers with its social investments and the total transparency of its financial actions.
\end{abstract}

Keywords: Ethical Banking; Traditional Banking; Social Banking; Social Economy and Social Values.

1 Universidad de Valencia, España

Dirección de correo electrónico: fcliment@uv.es

2 Universidad de Valencia, España

Dirección de correo electrónico: ma91vi@gmail.com 
Sumario. 1. Introducción. 2. La Banca Ética. 3. Banca Ética vs Banca Tradicional. 4. Conclusión. 5. Referencias bibliográficas.

Cómo citar: Climent Diranzo, F.J. y Escrivá Llidó, M.V. (2019) Banca Ética y Banca Tradicional. Comparativa entre Triodos Bank y Banco Santander. REVESCO. Revista de Estudios Cooperativos, Primer Cuatrimestre, $\mathrm{N}^{\mathrm{o}}$ 130, pp. 55-72. DOI: 10.5209/REVE.61935.

\section{Introducción}

A raíz de la crisis financiera iniciada en 2008, se generó una destacada desconfianza por parte de los ciudadanos con el sistema financiero que desembocó en una crisis ética (Camacho, Fernández, González y Miralles, 2013). Con el descontento de los ciudadanos, muchos de ellos empezaron a preocuparse por el destino que daban los bancos a sus ahorros y a interesarse en los valores éticos y sociales que ofrecía la Banca Ética (Soler y Melián, 2012; San José, Retolaza y Gutiérrez, 2011). Esta banca se diferencia de la Banca Tradicional porque no solo intenta obtener unos beneficios económicos, sino que también quiere tener un impacto social promoviendo la inversión hacia proyectos éticos (Cabaleiro y Rodríguez, 2008; Retolaza y San Emeterio, 2003; San José, Retolaza y Gutiérrez, 2011).

A partir de esta situación, se plantea la cuestión de si puede llegar a ser la Banca Ética más rentable que la Banca Tradicional invirtiendo únicamente en valores sociales. Con esta premisa, el trabajo plantea abordar los siguientes objetivos, en primer lugar, definir el concepto de Banca Ética, sus características y origen, en segundo lugar, analizar las entidades de Banca Ética que operan en España y, finalmente, realizar una comparativa entre Banca Ética y Banca Tradicional, centrando el análisis en un caso de estudio que compone una entidad representativa de cada tipo de banco.

Para poder dar respuesta a los objetivos planteados, por un lado, se ha estructurado el trabajo en los siguientes apartados, el primero de ellos se centra en analizar con detalle el origen y el concepto de Banca Ética. Además, también se describen algunas iniciativas de esta tipología de banca que operan en España como es el caso de Triodos Bank, Fiare Banca Ética y Coop 57, entre otros.

En el segundo apartado, se analizan las principales diferencias entre la Banca Tradicional y la Banca Ética. A continuación, se plantea un caso de estudio dónde se realiza una comparación en términos financieros entre un banco ético (Triodos Bank) y un banco tradicional (Banco Santander). Por último, se presentan las principales conclusiones alcanzadas.

\section{La Banca Ética}

\subsection{Concepto y Origen}

La Banca Ética surgió con la intención de institucionalizar las inversiones socialmente responsables. La inversión socialmente responsable combina los criterios éticos y los económicos para que las inversiones tengan en cuenta las 
condiciones financieras y la utilización de los fondos por parte de las entidades gestoras (San Emeterio y Retolaza, 2003 y Ruiz y Retolaza, 2007). Esto supuso la aparición de fondos de inversión responsables y el desarrollo del concepto de "Responsabilidad Social Corporativa". Este término incluye conceptos como el de ética bancaria, fondos éticos, inversión solidaria, responsabilidad social y banca ética (San Emeterio y Retolaza, 2003; Ruiz y Retolaza, 2007).

El término Banca Ética tiene múltiples definiciones, pero en este trabajo se van a destacar únicamente dos que se han considerado las más relevantes. La primera definición de Banca Ética es la que indica que esta banca está constituida por "un heterogéneo conjunto de entidades bancarias especializadas en la financiación de los colectivos más desfavorecidos, las empresas de la economía social, las ONG y las empresas más responsables con su entorno humano, social y ecológico" (Sasia y de la Cruz, 2008, p.30).

La segunda definición es la que aporta Alsina (2002, p.29), y que otros autores también hacen referencia (Cristianisme i Justícia, 2002; Ballesteros, 2003; Del Río, 2003; San Emeterio y Retolaza, 2003; Ruiz y Retolaza, 2007; Sasia y de la Cruz, 2008), se considera que el banco ético intenta conseguir simultáneamente financiar las actividades económicas que tengan un impacto social positivo y obtener un beneficio.

Asimismo, cabe destacar que la Banca Ética forma parte de las empresas solidarias, tal y como sugieren Ballesteros (2003) y Sanchis (2013), y por este motivo, los proyectos que financia la Banca Ética se basan en los siguientes principios:

Principio de igualdad: Se basa en el apoyo a proyectos y empresas que favorecen la participación activa de todos los miembros de la organización y sistemas de retribución con pocas diferencias salariales entre los directivos y los trabajadores.

- Principio de empleabilidad: Apoya los proyectos y empresas que favorezcan la generación de empleo estable y el acceso al empleo de los colectivos con mayor riesgo de exclusión.

- $\quad$ Principio de medioambiente: Se ha de apoyar proyectos que garanticen un crecimiento sostenible.

- Principio de cooperación: Apoyo de proyectos y empresas que tengan como referencia la cooperación interna (entre los empleados de la organización) y externa (con otras organizaciones).

Principio de compromiso con el entorno: Financiando proyectos y empresas que contribuyan al desarrollo de la comunidad local y de la sociedad en general, financiando actividades de dinamización de la sociedad.

- Principio de reinvertir en la sociedad parte de los beneficios financieros generados.

Estos principios definen a la Banca Ética y la diferencian de la Banca Convencional, convirtiéndose en un referente de empresa socialmente responsable, en un contexto en el que se ha considerado la banca convencional como especuladora, con falta de transparencia e incluso han realizado un abuso en las prácticas bancarias, como son las elevadas comisiones, desahucios, etc. 
Por tanto, las primeras experiencias de banca ética, tal y como indica Sanchis (2013), se pueden encontrar en los Montes de Piedad que surgieron en Europa en el siglo XV, concretamente en el año 1462. A partir de éstas, surgieron las cajas de ahorro en la segunda mitad del siglo XVIII que se dividieron en cooperativas de crédito y cajas de ahorro.

Así, en 1976 se creó el primer banco ético, el Grameen Bank en Bangladesh dedicado a ofrecer microcréditos a mujeres rurales sin recursos económicos. "Este banco y su creador, Muhammad Yunus, han sido el referente mundial de la banca ética, y a partir de él han ido surgiendo otras experiencias similares a lo largo de todo el mundo" (Sanchis, 2013, p.119). Entre los múltiples bancos que se crearon, destacan el Sewa Bank en la India, la Caisse Solidaire en Francia y el South Shore en Chicago.

Posteriormente, diversos autores (Del Río, 2003; San Emeterio y Retolaza, 2003; Ruiz y Retolaza, 2007 y Sanchis, 2013) enuncian diferentes bancos éticos que aparecieron en los años 80 como son Triodos Bank (Holanda), ABS (Suiza) y en los años 90, la Banca Popolare Etica (Italia), entre otros.

En España, en esta misma década, como afirman San Emeterio y Retolaza (2003) y Ruiz y Retolaza (2007), se produjo el primer intento de crear una banca alternativa, la Asociación para la Banca Ética y Ecológica (ABSE), que por distintos motivos desapareció. Después en 1990 resurgió con fuerza el interés por desarrollar las primeras experiencias de Banca Ética, coincidiendo este interés en el momento en que la banca convencional española comienza a transformarse en una banca especuladora (Sanchis, 2013).

En el año 1995 surgió la cooperativa de servicios financieros (Coop57), Oikocredit, Fundación de la Red de Economía Alternativa y Solidaria (REAS) e Iniciativas de Economía Alternativa y Solidaria (IDEAS). Además, en 1999 Caixa Colonya crea "L'estalvi Étic" la primera experiencia de banca ética que una institución financiera realiza en España. Estas iniciativas, según Del Río (2003) y Sanchis (2013), no son estrictamente bancos éticos, ya que no tienen la condición de banco reconocida por el Banco de España, aunque realizan una labor importante relacionada con la inversión socialmente responsable.

Estos mismos autores afirman que algunas cooperativas de crédito establecen acuerdos de cooperación con diferentes asociaciones y fundaciones vinculadas con la inversión socialmente responsable como la Caja Laboral Popular y REA en Navarra para la concesión de préstamos solidarios o la Caixa Popular y la Fundación Nova Terra en Valencia para el apoyo financiero a la creación de empresas de inserción. Otros autores (Del Río, 2003; San Emeterio y Retolaza, 2003 y Ruiz y Retolaza, 2007) ponen de manifiesto que en el año 2000 se pusieron en marcha otras iniciativas como la Creación de la Red de Útiles Financieros (RUFAS), el Financiamiento Ético y Solidario (FETS), EnClau, Fiare y el Proyecto Trust.

Hay que destacar que el Proyecto Trust, nació en 1998 en Madrid y tenía la intención de llegar a ser una banca social. Así, en 2003 consiguieron consolidar el acuerdo con Triodos Bank para constituir la primera banca social en España (Del 
Río, 2003 y San Emeterio y Retolaza, 2003). Por tanto, se puede concluir que en este año nace la Banca Ética en España. ${ }^{3}$

\subsection{Modelos que operan en España}

Una vez descritas las principales características y elementos diferenciadores de la Banca Ética, a continuación, se analizan algunas de las principales entidades de esta banca que operan en España, como son, Triodos Bank, Fiare Banca Ética y Coop 57.

Los orígenes de Triodos Bank (www.triodos.es), se remontan al año 1968 cuando un grupo de economistas, que formaban parte de la asociación Dinero Consciente, promovieron la idea de utilizar el dinero de forma ética y responsable. Posteriormente, en el año 1971 crearon la Fundación Triodos con el objetivo de captar fondos de donantes privados para invertirlos en actividades con fines sociales, medioambientales y culturales. En el año 1980, la Fundación Triodos dio lugar a la implantación de Triodos Bank en Holanda que ha sido el referente de la Banca ética en Europa y, actualmente, tiene sucursales en Holanda, Bélgica, Reino Unido, España y Alemania.

Como se ha indicado anteriormente, las primeras experiencias de Banca ética en España surgieron en el año 1998 con el Proyecto Trust que, a partir del año 2003, se convierte en Triodos Invest, siendo éste el intermediario financiero de Triodos Bank en España. En el 2004, fue cuando Triodos Bank consiguió operar como el primer Banco Social en España con ficha bancaria del Banco de España que la reconoce como tal.

Este banco nace con el objetivo de promover un cambio positivo en la sociedad desde el sistema financiero. Su intención es financiar a empresas y organizaciones que trabajan en el ámbito social, medioambiental y cultural, gracias al apoyo de ahorradores que optan por una sociedad más humana y sostenible. Triodos Bank, como describe Sanchis (2013, p.137), se caracteriza por tener la siguiente triple misión: "Contribuir a una sociedad que fomente la calidad de vida y se centre en la dignidad de las personas; facilitar que particulares, empresas y organizaciones realicen un uso responsable del dinero y fomentar con ello, el desarrollo sostenible; y proporcionar a sus clientes, productos financieros sostenibles y prestarles un servicio de calidad".

Destaca que es un banco que no cotiza en Bolsa con el objeto de no estar sometido a los vaivenes de precios que se producen en el mercado de capitales. De hecho, las acciones de la entidad están gestionadas por la Fundación para la Administración de las Acciones de Triodos Bank (SAAT). Además, según Abad y Valls (2018), en 2016, el patrimonio gestionado por el Grupo Triodos Bank era de 13.454 millones de euros, el $17 \%$ del total de los préstamos concedidos se realizan en la sucursal española y el número de clientes de banca ética ha aumentado en España un 48\% entre 2013 y 2016.

3 Para un mayor detalle de la evolución de la banca ética en España se puede consultar el trabajo de Abad y Valls (2018). 
Triodos Bank desarrolla un modelo de negocio bancario basado en los siguientes valores que suponen una base para cumplir nuestro objetivo de crear valor social desde el sistema financiero:

- Sostenibilidad: Eligen conceder financiación a empresas y actividades que beneficien a las personas, al medio ambiente y a la cultura. De esta forma, se promueve el desarrollo sostenible desde un sector clave como es la banca.

- Transparencia: Todos los clientes conocen las empresas y los proyectos que financian con sus ahorros e inversiones y les hacen partícipes del cambio que promueven con su dinero.

- Excelencia: Ofrecen una gran cantidad de productos y servicios financieros sostenibles y de calidad. Para conseguir que los clientes puedan operar de forma efectiva con Triodos Bank, como su primer banco.

- Iniciativa empresarial: Promueven la iniciativa empresarial mediante la financiación de sectores sostenibles e innovadores y también, el apoyo a emprendedores.

En cuanto a la fundación Fiare (http://www.fiarebancaetica.coop) nace en el 2003, con el fin de que los ciudadanos empezaran a conocer los cimientos de la Banca Ética. En el año 2005 se firmó el contrato de agentes con Banca Popolare Etica y en el año 2011, se integró Fiare y Banca Popolare Etica en España como una herramienta al servicio de la transformación social mediante la financiación de proyectos de la economía social y solidaria y la promoción de una cultura de la intermediación financiera. Fiare Banca Ética nace a partir de la fusión entre Fiare y Banca Popolare, llegando a tener actualmente, oficinas permanentes en Barcelona, Bilbao y Madrid.

Su misión es recuperar el valor social del dinero, es por ello, que los depósitos de ahorro sirven para poder financiar el desarrollo cooperativo, los valores transformadores, la agroecología, la cooperación al desarrollo, el comercio justo y la lucha contra la exclusión social.

Los principios en los que se basa Fiare Banca Ética son:

- Transparencia: Ofrece al público en general toda la información de sus actividades, inversiones, proyectos, entre otros.

- Participación: Es cuando los socios tienen la posibilidad, tanto de asistir a las juntas generales, como de tomar decisiones.

Democracia: Todos los socios tienen los mismos votos, ya que se basan en la regla "una persona, un voto" y es independiente del capital que hayan aportado.

- Derecho al crédito: Cualquier persona puede acceder a financiarse o a financiar su proyecto.

La participación y el principio de democracia son posibles gracias a que Fiare Banca Ética es un banco cooperativo. Además, también lo permite su estructura, ya que está basada en la participación de los socios que se organizan a nivel local con el objetivo de trabajar la actividad cultural, la interacción con la estructura operativa, la evaluación ético-social de las solicitudes de financiación e identificación de las necesidades del territorio en relación a los servicios bancarios. 
Sanchis y Pascual (2017) analizan las similitudes y diferencias entre los bancos cooperativos y los bancos éticos mediante la realización de un análisis de casos consistente en un estudio comparativo entre la cooperativa de crédito Caixa Popular y el banco ético Fiare Banca Ética. Del análisis se concluye que ambos modelos de banca social se basan en un sistema democrático y participativo en el que trabajadores y clientes participan activamente en su funcionamiento y ambos contribuyen a la inclusión financiera. En cambio, el modelo de la banca cooperativa es un modelo de proximidad basado en el trato personalizado y directo al cliente a través de las oficinas bancarias, mientras que el modelo de banca ética está basado en la banca electrónica con escasas sucursales.

Por último, en el año 1986 la empresa editorial Burguera tuvo que cerrar a causa de la crisis industrial. Muchos de los trabajadores de esta empresa, molestos por su situación, invirtieron sus indemnizaciones que recibieron por el despido y decidieron crear en 1995 la cooperativa "Coop 57" (https://coop57.coop/) en Cataluña.

Sanchis (2016), afirma que Coop 57 se creó bajo la forma jurídica de cooperativa de servicios financieros éticos y solidarios. Inicialmente, su desarrollo estaba muy vinculado al cooperativismo de trabajo asociado, y fue ampliando su base social a otro tipo de entidades de la economía social y solidaria a través de la intermediación financiera. Esto significa que recoge y capta ahorro de la sociedad civil para poder canalizarlo hacia la financiación de entidades de la economía social y solidaria que promueven la ocupación, el cooperativismo, el asociacionismo, la solidaridad y la sostenibilidad sobre la base de principios éticos y solidarios.

A partir del año 2005, Coop 57 puso en práctica un modelo de crecimiento en red. Este mismo año, una red de entidades de economía social y solidaria de Aragón y Coop 57, decidieron compartir el proyecto. Utilizando el modelo, la estructura jurídica y técnica de Coop 57, pero aplicando un modelo que permitiese que cada territorio se autogestionase. A partir de ello, surgió Coop 57 en Aragón (2005), Madrid (2006), Andalucía (2008) y Galicia (2009).

Los principios en los que se basa Coop 57 son:

- Coherencia: Evitan la contradicción de destinar dinero a actividades que no se correspondan con sus valores y principios éticos.

- Compatibilidad: Se compatibiliza el rendimiento financiero y el rendimiento social. No persiguen la maximización del beneficio económico, sino el máximo beneficio social ligado a la viabilidad financiera.

- Participación: Se fomenta la responsabilidad de los inversores ahorradores en sus operaciones financieras. Incentivando la participación de los socios en la política de la entidad, para así fomentar que recuperen el control de los ahorros y recursos económicos y promuevan la autogestión financiera.

Transparencia: Se aplica una absoluta transparencia en la gestión de la entidad, tanto en la concesión de préstamos, como en el funcionamiento interno y societario. Toda persona y entidad socia sabe para qué sirven los recursos que ha aportado. Se publican dos veces al año un boletín informativo donde se incluye la lista de préstamos acordados con cada entidad beneficiada, así como el volumen y la duración de cada préstamo. 
Solidaridad: Las personas y entidades socias de Coop57 saben que su dinero contribuye a construir una economía más humana y solidaria. Además, se puede manifestar, en la Asamblea, la solidaridad fijando la tasa de remuneración del dinero y el coste de los préstamos.

Reflexión: Se entiende el comportamiento ético como un proceso y se adaptan los valores y principios que se consideran justos y prioritarios. Se adaptan a las necesidades de las personas y entidades socias para contribuir a procesos de construcción alternativas.

\section{Banca Ética vs Banca Tradicional}

La principal diferencia entre Banca Ética y Banca Tradicional está en el objetivo de éstas, ya que, la Banca Ética se caracteriza por tener un objetivo social y económico, siendo éste último necesario para poder conseguir el primero. Sin embargo, la Banca Tradicional únicamente tiene un objetivo económico.

Esta característica de la Banca Ética se ve reflejada cuando, a la hora de operar, prefiere repartir a sus clientes una remuneración más baja e invertir el dinero en proyectos sociales. Además, tal y como indica Sandri $(2002$, p.94) “... los intereses que se dan a los clientes son más bajos que los del mercado, los intereses que se cobran no difieren demasiado con los de los bancos tradicionales". Con estas condiciones, los Bancos Éticos deberían tener mayores beneficios que la Banca Convencional, sin embargo, Alsina (2002) afirma que las ganancias de los Bancos Éticos son inferiores a las de las entidades tradicionales, por tres razones:

- Sin especulaciones: Se descartan todas aquellas inversiones que impliquen costes sociales y medioambientales y que sean muy rentables, como las inversiones especulativas en bolsa.

- Se necesita tiempo: Los Bancos Éticos suelen apostar por inversiones más a largo que a corto plazo. Consideran que los proyectos sociales necesitan un tiempo técnico para arrancar y por ello, el retorno de las inversiones no es inmediato y se diluye con el paso del tiempo.

- Costes adicionales: Para estos bancos conceder un crédito es caro, ya que los importes son reducidos y por tanto, el margen de ganancia también lo es. Hay más riesgos porque a la hora de conceder muchas de las financiaciones, no necesitan ninguna garantía real, además se preocupan por hacer un seguimiento técnico de los distintos proyectos (asesoramiento y formación), lo que conlleva un coste adicional.

Los clientes prefieren invertir en este tipo de banca, aunque no consigan la misma rentabilidad que en otros bancos, ya que éstos fomentan una mejora social. Además, estos clientes han perdido la confianza en la Banca Tradicional y por este motivo, prefieren invertir en la Banca Ética porque esta banca les ofrece transparencia y participación. En este sentido, la Banca Ética les permite elegir dónde invertir sus ahorros para darle un uso responsable. Además, cabe destacar que la Banca Ética se basa en el principio "una persona, un voto", por tanto, todos los miembros tienen los mismos derechos. Mientras que en la Banca Tradicional se 
rige por la regla "una acción, un voto", por lo que, cuantas más acciones más representación tiene.

Además, Ruíz y Retolaza (2007) indican que, aunque un banco haya utilizado ciertos criterios éticos, esto no lo ubica en el concepto de Banca Ética, ya que estos criterios solo suelen afectar a un fondo de inversión concreto y pueden coexistir con otros fondos de inversión que no tienen ninguna regularización ética. La Banca Tradicional utiliza estos criterios éticos como un instrumento de marketing con la intención de conseguir mayores beneficios.

Por este motivo, según Cowton y Thomson (1999, citado en Ruiz y Retolaza, 2007, p.1111), "las iniciativas de banca ética tienen el deseo de diferenciarse de la banca convencional" en este sentido, y aunque podamos clasificar a las diversas entidades bancarias en un continuo comportamiento ético, existe un punto de ruptura que diferencia a la Banca Ética de la Banca Tradicional.

Por último, hay que resaltar que la Banca Ética y la Banca Tradicional tienen diferentes finalidades, (Ballesteros, 2003). Por un lado, la Banca Tradicional ofrece productos atractivos para sus clientes e interesantes rendimientos a los accionistas que invierten su capital. Esta banca no reflexiona sobre la ideología del ahorro que capta de sus clientes, ni tampoco realiza estudios acerca del impacto social de los proyectos empresariales a los que presta el dinero, sino que solo intenta garantizar su devolución.

Por otro lado, la Banca Ética ofrece pocos productos y unos criterios de funcionamiento diferentes en comparación con la tradicional. Sus depósitos provienen de unas personas con una ideología determinada que quieren poner su dinero al servicio de proyectos y personas que no podrían entrar en los canales de la Banca Tradicional por su alto riesgo derivado de situaciones de exclusión. En la Tabla 1 se resumen las principales diferencias entre la Banca Ética y la Banca Tradicional. 
Tabla. 1. Diferencias entre la banca ética y tradicional.

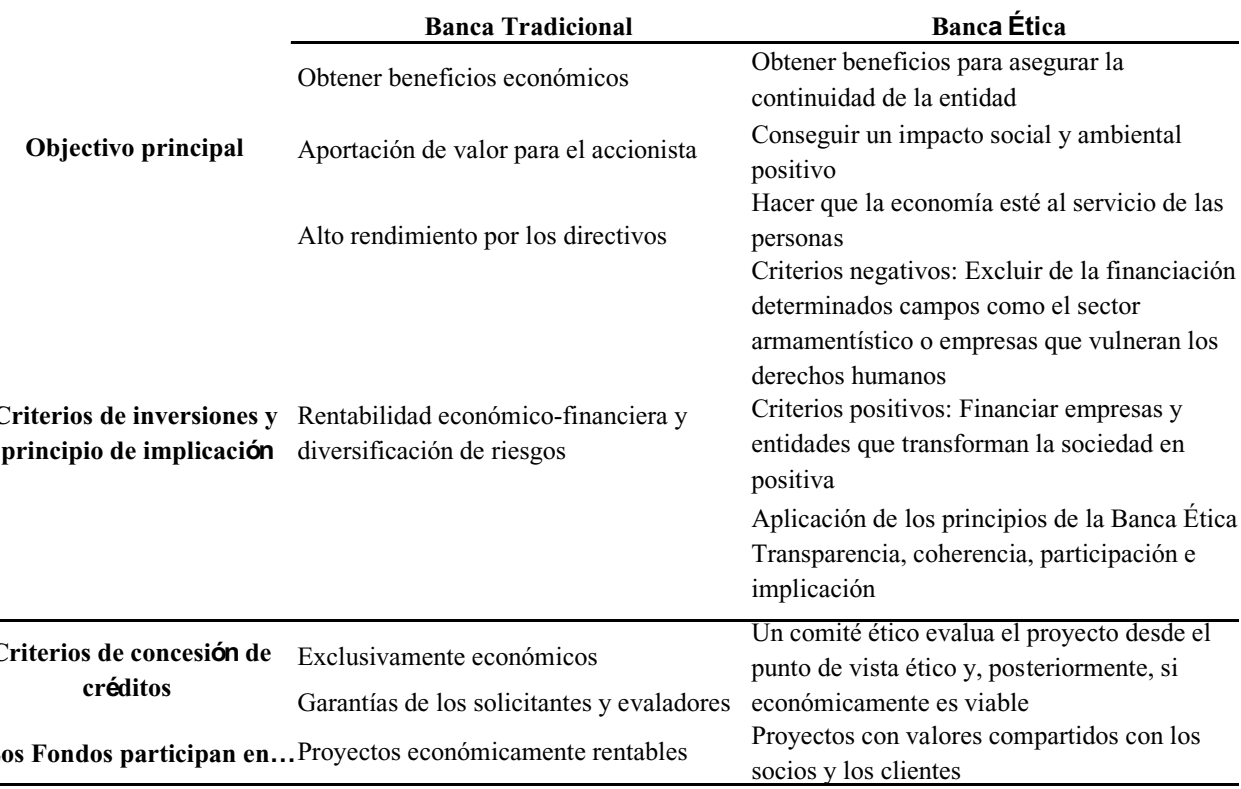

La mayoría de entidades financieras éticas $\begin{array}{lll}\text { Ánimo de lucro } & \begin{array}{l}\text { Su razón de ser es la remuneración del } \\ \text { accionista }\end{array} & \begin{array}{l}\text { no tienen ánimo de lucro, en estos casos los } \\ \text { beneficios revierten en proyectos sociales o }\end{array}\end{array}$

medio ambientales

\begin{tabular}{cll} 
Modelo de organización & Una acción equivale a un voto & $\begin{array}{l}\text { En los casos de entidades cooperativas (la } \\
\text { mayoría): una persona equivale a un voto }\end{array}$ \\
\hline \multirow{2}{*}{ Transparencia } & $\begin{array}{l}\text { No dan formación habitual y de manera } \\
\text { estable sobre los proyectos financiados }\end{array}$ & $\begin{array}{l}\text { Ofrecen información regular y pública de las } \\
\text { actividades financiadas }\end{array}$ \\
Coheréncia & $\begin{array}{l}\text { Financian proyectos que pueden no } \\
\text { coincidir con los valores de los clientes }\end{array}$ & $\begin{array}{l}\text { Inversiones en actividades de acuerdo con } \\
\text { los valores de los clientes }\end{array}$ \\
\hline \multirow{2}{*}{ Participación } & $\begin{array}{l}\text { Solo participan los acionistas y en función La mayoría de entidades animan y facilitan la } \\
\text { del capital aportado }\end{array}$ \\
\hline \multirow{2}{*}{ El cliente y su dinerocipación de sus socios }
\end{tabular}

Fuente: www.bancaetica.cat/es/index.php (fecha de consulta, mayo 2017).

\subsection{Análisis económico}

Con el objeto de comprobar si la Banca Ética es más rentable que la Banca Tradicional, se realiza un análisis económico centrado en la actividad financiera de ambas tipologías de bancos a lo largo de 7 años (2011-2017), a partir de sus balances de situación y las cuentas de resultados anuales obtenidos de la Base de datos Orbis (https://www.bvdinfo.com).

Para ello, se ha escogido por parte de la Banca Tradicional, al Banco Santander por ser el de mayor Volumen de Activos de España, y por parte de la Banca Ética se ha elegido a Triodos Bank, por ser la Banca Ética de mayor tamaño en términos 
relativos en España. Para realizar este análisis hay que tener en cuenta que el tamaño del Banco Santander es mucho mayor al de Triodos Bank y por este motivo todas las cifras que se utilizan se expresan en términos relativos, para así poder ver su evolución a lo largo del período de estudio.

Para llevar a cabo este análisis, se analiza el Balance, la Cuenta de Pérdidas y Ganancias y las Ratios de Liquidez, Endeudamiento y Rentabilidad de ambos bancos. También se compara en términos relativos el Número de Empleados, el Beneficio que estos aportan, el Volumen de Créditos y el de Depósitos.

\subsubsection{Análisis preliminar}

Según la Tabla 2, durante el periodo 2011-2017 el número de empleados de Triodos Bank ha aumentado un 91\% mientras que el Banco Santander únicamente un 5\%. Además, con respecto al beneficio por empleado el crecimiento de esta ratio ha sido superior en el caso del Banco Santander, aunque en los años 2012, 2013 y 2015 la ratio ha sido superior en Triodos Bank.

En cuanto al Volumen de Créditos que han concedido ambas entidades durante el período analizado, en el caso de Triodos Bank se ha incrementado en un $132 \%$ frente al 13\% del Banco Santander.

Por lo que respecta al Volumen de Depósitos que los clientes han contratado durante el período analizado, se comprueba que éste ha aumentado en ambas entidades, siendo muy superior en el caso de Triodos Bank se sitúa (134\%), frente al del Banco Santander (22\%). Esta evolución pone de manifiesto que los clientes han decidido empezar a apostar por la Banca Ética, ya que el incremento en términos relativos ha sido mucho mayor en Triodos Bank en lo que respecta al Volumen de créditos y de depósitos.

Tabla. 2. Comparativa del número de empleados, el beneficio por empleado, el volumen de depósitos y de créditos en millones de $€$.

\begin{tabular}{|c|c|c|c|c|c|c|c|c|c|}
\hline & Entidades & 2011 & 2012 & 2013 & 2014 & 2015 & 2016 & 2017 & $\begin{array}{l}\text { Diferencia } \\
\text { Porcentual } \\
\text { (2011- } \\
\text { 2017) } \\
\end{array}$ \\
\hline \multirow{2}{*}{$\begin{array}{l}\text { Número de } \\
\text { empleados }\end{array}$} & $\begin{array}{l}\text { Triodos } \\
\text { Bank }\end{array}$ & 720 & 788 & 790 & 888 & 979 & 1,271 & 1,377 & $91 \%$ \\
\hline & $\begin{array}{l}\text { Banco } \\
\text { Santander }\end{array}$ & 187.233 & 188.779 & 186.373 & 183.938 & 193.863 & 191.635 & 195.732 & $5 \%$ \\
\hline \multirow{2}{*}{$\begin{array}{l}\text { Beneficio } \\
\text { por } \\
\text { empleado }\end{array}$} & $\begin{array}{l}\text { Triodos } \\
\text { Bank }\end{array}$ & 0.0319 & 0.0393 & 0.0430 & 0.0450 & 0.0552 & 0.0307 & 0.0363 & $14 \%$ \\
\hline & $\begin{array}{l}\text { Banco } \\
\text { Santander }\end{array}$ & 0,0424 & 0,0188 & 0,0411 & 0,0581 & 0,0492 & 0,0562 & 0,0618 & $46 \%$ \\
\hline \multirow{2}{*}{$\begin{array}{l}\text { Volumen } \\
\text { de } \\
\text { depósitos }\end{array}$} & $\begin{array}{l}\text { Triodos } \\
\text { Bank }\end{array}$ & 3.731 & 4.594 & 5.650 & 6.289 & 7.283 & 8.025 & 8.722 & $134 \%$ \\
\hline & $\begin{array}{l}\text { Banco } \\
\text { Santander }\end{array}$ & 588.977 & 589.104 & 572.853 & 608.956 & 647.578 & 657.770 & 720.606 & $22 \%$ \\
\hline
\end{tabular}




\begin{tabular}{llllllllll} 
Volumen & $\begin{array}{l}\text { Triodos } \\
\text { Bank }\end{array}$ & 2.838 & 3.285 & 3.545 & 4.266 & 5.216 & 5.708 & 6.598 & $132 \%$ \\
de créditos & Banco & & & & & & & & \\
& Santander & 742.044 & 711.320 & 663.777 & 731.790 & 784.767 & 780.966 & 840.100 & $13 \%$ \\
\hline
\end{tabular}

Fuente: Elaboración propia a partir de la base de datos Orbis

\subsubsection{Balance de Situación}

En primer lugar, se analizan ciertas partidas relevantes del Activo (Tabla 3). Los Activos líquidos de Triodos Bank se han incrementado en un 118\%, mientras que en el Banco Santander solo lo hacen en un 26\%. En cuanto a los de préstamos concedidos y fallidos destaca el crecimiento en el caso de Triodos Bank de un $132 \%$ y un $36 \%$, frente al $13 \%$ y $26 \%$ del Banco Santander, respectivamente.

En cuanto al Activo Total, durante el periodo 2011-2017 se incrementa un 15\%, en el caso del Banco Santander, frente a un 131\% en el caso de Triodos Bank, constatado el elevado ritmo de actividad de esta entidad en los últimos años.

Tabla. 3. Comparativa del Activo en millones de $€$

\begin{tabular}{|c|c|c|c|c|c|c|c|c|c|}
\hline & Entida des & 2011 & 2012 & 2013 & 2014 & 2015 & 2016 & 2017 & $\begin{array}{l}\text { Diferencia } \\
\text { Porcentual } \\
(2011-2017)\end{array}$ \\
\hline \multirow{2}{*}{$\begin{array}{l}\text { Activos } \\
\text { Líquidos }\end{array}$} & $\begin{array}{l}\text { Triodos } \\
\text { Bank }\end{array}$ & 737 & 942 & 1,447 & 751 & 831 & 1,200 & 1,609 & $118 \%$ \\
\hline & $\begin{array}{l}\text { Banco } \\
\text { Santander }\end{array}$ & 223.708 & 261.350 & 215.581 & 233.976 & 243.805 & 243.245 & 276.905 & $24 \%$ \\
\hline \multirow{2}{*}{ Préstamos } & $\begin{array}{l}\text { Triodos } \\
\text { Bank }\end{array}$ & 2.838 & 3.285 & 3.545 & 4.266 & 5.216 & 5.708 & 6.598 & $132 \%$ \\
\hline & $\begin{array}{l}\text { Banco } \\
\text { Santander }\end{array}$ & 742.044 & 711.320 & 663.777 & 731.790 & 784.767 & 780.966 & 840.100 & $13 \%$ \\
\hline \multirow{2}{*}{$\begin{array}{l}\text { Préstamos } \\
\text { fallidos }\end{array}$} & $\begin{array}{l}\text { Triodos } \\
\text { Bank }\end{array}$ & 36 & 55 & 61 & 67 & 67 & 56 & 49 & $36 \%$ \\
\hline & $\begin{array}{l}\text { Banco } \\
\text { Santander }\end{array}$ & 18.465 & 24.621 & 24.002 & 26.241 & 25.650 & 23.654 & 23.271 & $26 \%$ \\
\hline \multirow{2}{*}{$\begin{array}{l}\text { Activo } \\
\text { Total }\end{array}$} & $\begin{array}{l}\text { Triodos } \\
\text { Bank }\end{array}$ & 4.291 & 5.291 & 6.447 & 7.152 & 8.211 & 9.081 & 9.902 & $131 \%$ \\
\hline & $\begin{array}{l}\text { Banco } \\
\text { Santander }\end{array}$ & 1.251 .526 & 1.269 .628 & 1.115 .638 & 1.266 .296 & 1.340 .260 & 1.339 .125 & 1.444 .305 & $15 \%$ \\
\hline
\end{tabular}

Fuente: Elaboración propia a partir de la base de datos Orbis

En segundo lugar, en la Tabla 4, se analiza el Patrimonio Neto que está compuesto por las aportaciones que han realizado los socios tanto para constituir la sociedad como en las ampliaciones de Capital que se han realizado. Así mismo, 
también está formado por la dotación de reservas que han destinado las entidades y el resultado de ejercicios anteriores.

Así, en el período analizado el Patrimonio Neto ha crecido un $125 \%$ para Triodos Bank y un 23\% en el caso del Banco Santander. En cualquier caso, al no disponer de suficiente patrimonio neto, ambas entidades recurren a la financiación externa para hacer frente a su activo.

Además, se analiza el Pasivo Total y los Depósitos y Financiación a corto plazo, donde se observa en ambas entidades que la principal fuente de financiación se centra en el corto plazo, pero con un crecimiento muy superior, en ambas partidas, por parte de Triodos Bank.

Tabla. 4. Comparativa del Patrimonio Neto y Pasivo en millones de $€$

\begin{tabular}{|c|c|c|c|c|c|c|c|c|c|}
\hline & Entida des & 2011 & 2012 & 2013 & 2014 & 2015 & 2016 & 2017 & $\begin{array}{l}\text { Diferencia } \\
\text { Porcentual } \\
\text { (2011- } \\
\text { 2017) }\end{array}$ \\
\hline \multirow{2}{*}{$\begin{array}{l}\text { Patrimonio } \\
\text { Neto }\end{array}$} & $\begin{array}{l}\text { Triodos } \\
\text { Bank }\end{array}$ & 452 & 566 & 655 & 705 & 784 & 907 & 1.017 & $125 \%$ \\
\hline & $\begin{array}{l}\text { Banco } \\
\text { Santander }\end{array}$ & 98.431 & 97.198 & 94.377 & 105.090 & 113.247 & 117.158 & 121.322 & $23 \%$ \\
\hline \multirow{2}{*}{$\begin{array}{l}\text { Depósitos y } \\
\text { financiació } \\
\text { n a CP }\end{array}$} & $\begin{array}{l}\text { Triodos } \\
\text { Bank }\end{array}$ & 3.766 & 4.656 & 5.712 & 6.343 & 7.322 & 8.056 & 8.786 & $133 \%$ \\
\hline & $\begin{array}{l}\text { Banco } \\
\text { Santander }\end{array}$ & 750.799 & 793.487 & 712.162 & 748.876 & 856.034 & 858.839 & 936.276 & $25 \%$ \\
\hline \multirow{2}{*}{$\begin{array}{l}\text { Pasivo } \\
\text { Total }\end{array}$} & $\begin{array}{l}\text { Triodos } \\
\text { Bank }\end{array}$ & 3.839 & 4.725 & 5.792 & 6.447 & 7.427 & 8.174 & 8.885 & $131 \%$ \\
\hline & $\begin{array}{l}\text { Banco } \\
\text { Santander }\end{array}$ & 1.153 .095 & 1.172 .430 & 1.021 .261 & 1.161 .206 & 1.227 .013 & 1.221 .967 & 1.322 .983 & $15 \%$ \\
\hline
\end{tabular}

Fuente: Elaboración propia a partir de la base de datos Orbis

Además, se analiza la Ratio de Garantía que muestra la capacidad que aportan los activos para hacer frente a las deudas y se calcula dividiendo el Activo Total entre el Pasivo Total. Tal y como se observa en la Tabla 5, ambos bancos ofrecen garantía para hacer frente a sus deudas, ya que obtienen una ratio superior a 1 a lo largo del período analizado y con leves variaciones.

En cuanto a la Ratio de Liquidez, que nos indica la capacidad que tiene la empresa para generar recursos líquidos suficientes para atender a sus compromisos de pago a corto plazo y se calcula dividiendo el Activo Líquido entre los Depósitos y la Financiación a corto plazo, tanto el Banco Santander como Triodos Bank presentan una ratio positiva, aunque mayor sistemáticamente en el caso del Banco Santander. Por tanto, se puede concluir que ambos son capaces de cubrir sus deudas a corto plazo durante todo el período. Si bien, en términos relativos, esta ratio ha disminuido en ambos casos a lo largo del periodo analizado, especialmente en el caso de Triodos Bank (6.49\%). 
En lo que se refiere la Ratio de Morosidad, calculado como la relación entre préstamos fallidos y totales, se observa que para Triodos Bank en ningún caso se supera el $2 \%$, con un descenso del $41 \%$ en el periodo analizado. Mientras que en el caso de Banco Santander esta ratio se encuentra en un rango entre el $2.49 \%$ y el $3.62 \%$, con un crecimiento del $11.32 \%$ en el intervalo considerado.

Para analizar en profundidad la deuda total que poseen ambas entidades se estudia la Ratio de Endeudamiento Total, calculada como cociente entre el total del Pasivo y el Patrimonio Neto. Se observa que Triodos Bank presenta mayor endeudamiento, en el entorno del 8\%-9\%, con un incremento de casi el 3\% en el periodo considerado. Mientras que en el caso del Banco Santander se sitúa en el rango $10 \%-12 \%$, pero con un descenso de casi un $7 \%$ para el mismo intervalo.

Tabla. 5. Ratios relevantes

\begin{tabular}{|c|c|c|c|c|c|c|c|c|c|}
\hline & Entida des & 2011 & 2012 & 2013 & 2014 & 2015 & 2016 & 2017 & $\begin{array}{l}\text { Diferencia } \\
\text { Porcentual } \\
\text { (2011- } \\
\text { 2017) }\end{array}$ \\
\hline \multirow{2}{*}{$\begin{array}{l}\text { Ratio } \\
\text { Garantía }\end{array}$} & $\begin{array}{l}\text { Triodos } \\
\text { Bank }\end{array}$ & 1,12 & 1,12 & 1,11 & 1,11 & 1,11 & 1,11 & 1,11 & $-0.28 \%$ \\
\hline & $\begin{array}{l}\text { Banco } \\
\text { Santander }\end{array}$ & 1,09 & 1,08 & 1,09 & 1,09 & 1,09 & 1,10 & 1,09 & $0.58 \%$ \\
\hline \multirow{2}{*}{$\begin{array}{l}\text { Ratio } \\
\text { Liquidez }\end{array}$} & $\begin{array}{l}\text { Triodos } \\
\text { Bank }\end{array}$ & 19,58 & 20,22 & 25,34 & 11,84 & 11,35 & 14,89 & 18,31 & $-6.49 \%$ \\
\hline & $\begin{array}{l}\text { Banco } \\
\text { Santander } \\
\end{array}$ & 29,80 & 32,94 & 30,27 & 31,24 & 28,48 & 28,32 & 29,58 & $-0.74 \%$ \\
\hline \multirow{2}{*}{$\begin{array}{ll}\text { Ratio de } \\
\text { Morosidad }\end{array}$} & $\begin{array}{l}\text { Triodos } \\
\text { Bank }\end{array}$ & 1,27 & 1,68 & 1,72 & 1,56 & 1,29 & 0,99 & 0,75 & $-41.38 \%$ \\
\hline & $\begin{array}{l}\text { Banco } \\
\text { Santander }\end{array}$ & 2,49 & 3,46 & 3,62 & 3,59 & 3,27 & 3,03 & 2,77 & $11.32 \%$ \\
\hline \multirow{2}{*}{$\begin{array}{l}\text { Endeudamiento } \\
\text { Total }\end{array}$} & $\begin{array}{l}\text { Triodos } \\
\text { Bank }\end{array}$ & 8,50 & 8,35 & 8,84 & 9,15 & 9,47 & 9,01 & 8,73 & $2.73 \%$ \\
\hline & $\begin{array}{l}\text { Banco } \\
\text { Santander }\end{array}$ & 11,71 & 12,06 & 10,82 & 11,05 & 10,83 & 10,43 & 10,90 & $-6.91 \%$ \\
\hline
\end{tabular}

Fuente: Elaboración propia a partir de la base de datos Orbis

\subsubsection{Cuenta de Pérdidas y Ganancias}

Para analizar la cuenta de Pérdidas y Ganancias se estudian los ingresos, los gastos, los impuestos sobre beneficios y el resultado final, (Tabla 6). Así, con respecto a los ingresos, Triodos Bank constata un incremento del $88 \%$ mientras que el Banco Santander únicamente del $10 \%$. Por el contrario, en lo que respecta a los gastos, Triodos Bank presenta un incremento del $81 \%$ mientras que el Banco Santander ha tenido un incremento de un $1 \%$. Estos resultados ponen de manifiesto que la banca tradicional, durante el periodo analizado, ha llevado a cabo un importante proceso 
de reestructuración materializado en cierre de oficinas, reducción de personal y, en general, una mayor mejora sustancial de su eficiencia.

En cuanto a los impuestos sobre beneficios, en ambas entidades financieras han ascendido durante el período analizado, constatando una mejora en los resultados. Así, Triodos Bank crece un 100\% mientras que el Banco Santander asciende un 119\%.

Por último, se analiza el resultado del ejercicio obtenido después de aplicarle los impuestos al resultado bruto, destacando que el resultado de Triodos Bank se ha incrementado un 124\% mientras que el Banco Santander lo ha hecho en un 33\%. En definitiva, se pone de manifiesto que el crecimiento de los beneficios de la Banca Ética es superior, a pesar de tener que financiar proyectos sociales y soportar, en general, mayores costes.

Tabla. 6. Comparativa de los ingresos, gastos, impuestos y resultado del ejercicio en millones de $€$

\begin{tabular}{|c|c|c|c|c|c|c|c|c|c|}
\hline & Entida des & 2011 & 2012 & 2013 & 2014 & 2015 & 2016 & 2017 & $\begin{array}{l}\text { Diferencia } \\
\text { Porcentual } \\
\text { (2011- } \\
\text { 2017) }\end{array}$ \\
\hline \multirow{2}{*}{ Ingresos } & $\begin{array}{l}\text { Triodos } \\
\text { Bank }\end{array}$ & 129 & 152 & 164 & 190 & 212 & 218 & 242 & $88 \%$ \\
\hline & $\begin{array}{l}\text { Banco } \\
\text { Santander }\end{array}$ & 45.754 & 46.190 & 41.264 & 44.590 & 48.130 & 46.192 & 50.321 & $10 \%$ \\
\hline \multirow{2}{*}{ Gastos } & $\begin{array}{l}\text { Triodos } \\
\text { Bank }\end{array}$ & 106 & 121 & 130 & 150 & 158 & 179 & 192 & $81 \%$ \\
\hline & $\begin{array}{l}\text { Banco } \\
\text { Santander }\end{array}$ & 37.815 & 42.641 & 33.612 & 33.911 & 38.583 & 35.424 & 38.230 & $1 \%$ \\
\hline \multirow{2}{*}{ Impuestos } & $\begin{array}{l}\text { Triodos } \\
\text { Bank }\end{array}$ & 6 & 8 & 9 & 10 & 13 & 9 & 12 & $100 \%$ \\
\hline & $\begin{array}{l}\text { Banco } \\
\text { Santander }\end{array}$ & 1.776 & 575 & 2.113 & 3.718 & 2.213 & 3.282 & 3.884 & $119 \%$ \\
\hline \multirow{2}{*}{ Resultado } & $\begin{array}{l}\text { Triodos } \\
\text { Bank }\end{array}$ & 17 & 23 & 25 & 30 & 41 & 30 & 38 & $124 \%$ \\
\hline & $\begin{array}{l}\text { Banco } \\
\text { Santander }\end{array}$ & 6.163 & 2.974 & 5.539 & 6.961 & 7.334 & 7.486 & 8.207 & $33 \%$ \\
\hline
\end{tabular}

Fuente: Elaboración propia a partir de la base de datos Orbis

\subsubsection{Análisis de Rentabilidad}

Finalmente, se analiza tanto la rentabilidad económica como la financiera (Tabla 7). Por una parte, la Rentabilidad Económica (ROA), rentabilidad antes de descontar la retribución de los recursos utilizados y el impuesto sobre beneficios, en ambas entidades financieras se constata una tendencia creciente, siendo de un $10 \%$ para Triodos Bank y de un 18\% en el caso del Banco Santander. 
En cuanto a la Rentabilidad Financiera (ROE), rentabilidad que va dirigida exclusivamente a los accionistas y en la que influye la forma de financiación, Triodos Bank presenta un crecimiento de un $16 \%$, mientras que en el Banco Santander crece un $10 \%$, aunque con una rentabilidad financiera que en determinados años $(2011,2014,2016$ y 2017) ha duplicado la de Triodos Bank. Esta diferencia de crecimiento a favor del Banco Santander se puede justificar en el objetivo financiero de ambas entidades, mientras que el Banco Santander maximiza el beneficio, Triodos Bank se centra en financiar inversiones que tengan un impacto social positivo, ello repercute en una menor rentabilidad. Abad y Valls (2018), a partir de los informes anuales, obtienen resultados similares incluyendo en el análisis, además de Triodos Bank y Banco Santander, a la entidad BBVA durante el periodo 2014-2016.

Tabla. 7. Comparativa de las rentabilidades económicas y financieras

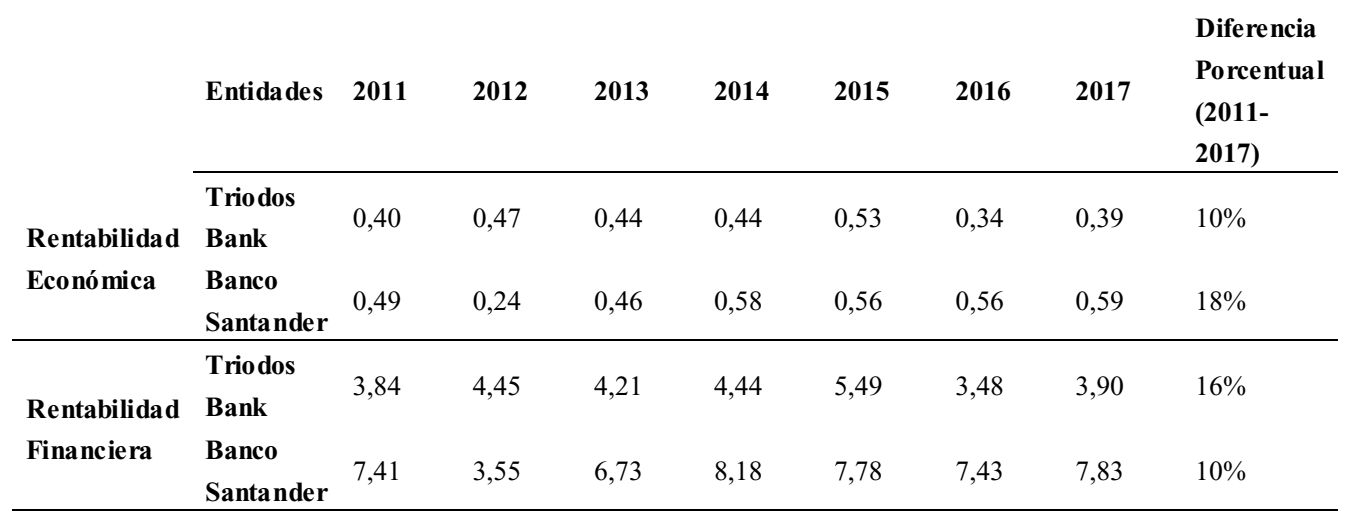

Fuente: Elaboración propia a partir de la base de datos Orbis

\section{Conclusión}

El objetivo de este trabajo es dar una respuesta a la pregunta inicial de si la Banca Ética es igual de rentable que la Banca Tradicional, y la conclusión alcanzada indica que la Banca Ética claramente es menos rentable por su inversión social.

Del análisis, se puede concluir que para el período 2011-2017, Triodos Bank ha tenido mayor incremento en cuanto al número de empleados que el Banco Santander. Esto ocurre debido a que Triodos Bank se encuentra en un proceso de expansión, mientras que el Banco Santander ha ido reduciendo su sobredimensionada red de sucursales y empleados.

Se observa un mayor incremento del volumen de créditos y de depósitos por parte de Triodos Bank, indicativo de que ha habido un crecimiento destacado del número de clientes que han decidido apostar por la Banca Ética, sacrificando parte de la rentabilidad de sus inversiones a cambio de invertir en proyectos más sociales. 
Además, destaca la significativa reducción de morosidad por parte de Triodos Bank, indicativo de que los clientes con perfiles más éticos se asocian con una menor probabilidad de incumplir sus compromisos financieros.

Con respeto al resultado del ejercicio, Triodos Bank obtiene un menor beneficio que el Banco Santander debido a que asume unos costes mayores al financiar proyectos sociales.

Por lo que respecta a su rentabilidad, es superior en el caso del Banco Santander debido a que su principal objetivo se centra en maximizar su beneficio, mientras que Triodos Bank concentra su inversión en proyectos sociales y medioambientales. Esta diferencia es primordial, ya que cada vez más ciudadanos buscan un banco que fomente la transparencia y unos valores beneficiosos para la sociedad.

En conclusión, tal y como afirma Sanchis (2013, p.149) "necesitamos una banca que utilice el dinero como instrumento al servicio de la sociedad y no como medio de pago al servicio de los poderosos, que financie proyectos éticos que posibiliten el desarrollo de los derechos humanos y no su destrucción".

\section{Referencias bibliográficas}

Abad, E. y Valls, M. C. (2018) Análisis de viabilidad de la banca ética en España a través de Triodos Bank. Comparativa económico-financiera con la banca tradicional. REVESCO. Revista de Estudios Cooperativos, Segundo Cuatrimestre, №. 128, pp. 1-29. DOI: 10.5209/REVE.60205.

Alsina, O. (2002) Banca Ética: mucho más que dinero. Barcelona: Icaria.

Ballesteros, C. (2003) La banca ética. En D. García, E. González, C. Ballesteros, y F. Navarro, La empresa socialmente responsable. Ética y empresa. Madrid: Cideal.

Cabaleiro, M. J. y Rodríguez, S. M. (2008) Sociedades cooperativas de banca alternativa. REVESCO. Revista de Estudios Cooperativos, Segundo Cuatrimestre, № 95, pp. 44-64.

Camacho, I., Fernández, J. L., González, R. y Miralles, J. (2013) Ética y Responsabilidad Empresarial. Bilbao: Desclee de Brower.

COOP 57. (s.f.) Coop 57. Recuperado 5 de mayo de 2016, de https://coop57.coop/.

Cristianisme i Justícia (2002) ¿Que hacen con mi dinero? Banca Ética: Mucho más que dinero. Cristianisme i Justícia, $\mathrm{N}^{\circ} 104$.

Del Río, N. (2003) Rescata tu dinero. Finanzas solidarias y transformación social. Madrid: Talasa.

FETS. (s.f.) Financiación Ética y Solidaria. Recuperado 10 de mayo de 2016, de http://fets.org/es/.

FIARE Banca Ética (s.f.) Recuperado 7 de mayo de 2016, de http://www.fiarebancaetica.coop/.

Gutiérrez, I. (2015) Informe de auditoría y cuentas anuales 2014 del Banco Santander, Madrid.

Retolaza, J. L. y San Emeterio, J. (2003) ¿Existe espacio para una banca ética? Revista de relaciones laborales, $\mathrm{N}^{\circ} .9$, pp. 127-163.

Ruiz, L. S., y Retolaza, J. L. (2007) Análisis comparativa de la Banca Ética con la Banca Tradicional: Identificación de indicadores. En J. C. Ayala (Coord.), Conocimiento, innovación y emprendedores: Camino al futuro, España: Universidad de La Rioja. 
San Emeterio, J., y Retolaza, J. L. (2003) ¿Existe Espacio para una Banca Ética? Lan Harremanak: Revista de relaciones laborales, № 9, pp. 127-163.

San José, L.; Retolaza, J. L. y Gutiérrez, J. (2011) Are Ethical Banks Different? A Comparative Analysis Using the Radical Affinity Index. Journal of Business Ethics, $\mathrm{N}^{\circ}$ 1, Vol. 100, pp. 151-173.

Sanchis, J. R. y Pacual, E. (2017) Banca ética y banca cooperativa. Un análisis comparativo a través del estudio de Caixa Popular y de Fiare Banca Ética. REVESCO. Revista de Estudios Cooperativos, Segundo Cuatrimestre, $\mathrm{N}^{\mathrm{o}}$ 124, pp. 152-174. DOI: 10.5209/REVE.54921.

Sanchis, J. R. (2013) La banca que necesitamos. Valencia: Publicacions de la Universitat de València.

Sanchis, J. R. (2016) ¿Es posible un mundo sin bancos? Barcelona: El Viejo Topo.

Sandri, P. (2002) Dinero ético. La economía alternativa y solidaria. Barcelona: Debolsillo.

Sasia, P., y De la Cruz, C. (2008) La Banca Ética y Ciudadania. Madrid: Trotta.

Soler, F. y Melián, A. (2012) Cooperativas de crédito y banca social: viejas y nuevas respuestas éticas y solidarias a problemas de siempre. REVESCO. Revista de Estudios Cooperativos, Tercer Cuatrimestre, $\mathrm{N}^{\mathrm{o}} 109$, pp. 45-80. DOI: 10.5209/rev_REVE.2012.v109.40655. 